\title{
NILPOTENTS AND UNITS IN SKEW POLYNOMIAL RINGS OVER COMMUTATIVE RINGS
}

\author{
M. RIMMER and K. R. PEARSON
}

(Received 3 April 1979)

Communicated by D. E. Taylor

\begin{abstract}
Let $R$ be a commutative ring with an automorphism $\alpha$ of finite order $n$. An element $f$ of the skew polynomial ring $R[x, \alpha]$ is nilpotent if and only if all coefficients of $f^{n}$ are nilpotent. (The case $n=1$ is the well-known description of the nilpotent elements of the ordinary polynomial ring $R[x]$.) A characterization of the units in $R[x, \alpha]$ is also given.
\end{abstract}

1980 Mathematics subject classification (Amer. Math. Soc.): 16 A 05.

Keywords: Skew polynomial, nilpotent, unit.

Let $R$ be a commutative ring and suppose $\alpha$ is an automorphism of $R$ with finite order $n$. We describe in Theorem 1 the nilpotent elements of $R[x, \alpha]$ in a way which is a generalization of the well-known characterization of the nilpotent elements in the ordinary polynomial ring $R[x]$ (the case $n=1$ ). In Section 2 we characterize the units in $R[x, \alpha]$. The results are all obtained by embedding $R[x, \alpha]$ into an $n \times n$ matrix ring.

We write the elements of $R[x, \alpha]$ in the form

$$
r_{0}+r_{1} x+\ldots+r_{m} x^{m} \quad\left(r_{i} \in R\right)
$$

and multiplication is determined by $x r=r^{\alpha} x$ for $r \in R$.

These results appear in the first author's Ph.D. thesis (Rimmer (1978)), written under the supervision of the second author. Gilmer (1975) describes the related results for $R[x]$. 


\section{Nilpotents}

THEOREM 1. Let $R$ be a commutative ring with an automorphism $\alpha$ of order $n$. An element $f$ of $R[x, \alpha]$ is nilpotent if and only if all coefficients of $f^{n}$ are nilpotent in $R$.

Proof. When $R$ is embedded in a ring with identity in the usual way, the automorphism $\alpha$ extends to an automorphism of the same order. Thus we may assume in what follows that $R$ has an identity.

Denote $x^{n}$ by $y$. Notice that $y$ is central in $R[x, \alpha]$ since

$$
y r=x^{n} r=r^{\alpha^{n}} x^{n}=r x^{n}=r y,
$$

that the subring $R[y]$ generated by $R$ and $y$ is just the ordinary polynomial ring and that $R[x, \alpha]$ is a free (left) $R[y]$-module with basis $\mathscr{B}=\left\{1, x, \ldots, x^{n-1}\right\}$. The regular representation ( $f$ maps to right multiplication by $f$ ) embeds $R[x, \alpha]$ in $\operatorname{End}_{R[y\}}(R[x, \alpha])$. If we replace elements of $\operatorname{End}_{R\{y\}}(R[x, \alpha])$ by their matrices with respect to $\mathscr{B}$ we have an embedding $\varphi$ from $R[x, \alpha]$ into the ring $M(n, R[y])$ of $n \times n$ matrices over $R[y]$. It is easy to check that if $h=h_{0}+h_{1} x+\ldots+h_{n-1} x^{n-1}$ (with $h_{i}$ in $R[y]$ ) then $h \varphi$ is the matrix

$$
\left(\begin{array}{ccccc}
h_{0} & h_{1} & h_{2} & \ldots & h_{n-1} \\
h_{n-1}^{\alpha} y & h_{0}^{\alpha} & h_{1}^{\alpha} & \ldots & h_{n-2}^{\alpha} \\
h_{n-2}^{\alpha^{2}} y & h_{n-1}^{\alpha^{2}} y & h_{0}^{\alpha^{2}} & \ldots & h_{n-3}^{\alpha^{2}} \\
\vdots & \vdots & \vdots & \ldots & \vdots \\
h_{1}^{\alpha^{n-1}} y & h_{2}^{\alpha^{n-1}} y & h_{3}^{\alpha^{n-1}} y & \ldots & h_{0}^{\alpha^{n-1}}
\end{array}\right) .
$$

Let $P$ be any prime ideal of $R$. The natural map $R \rightarrow R / P$ extends to a homomorphism

$$
\theta_{P}: M(n, R[y]) \rightarrow M(n,(R / P)[y]) .
$$

If $h=h_{0}+h_{1} x+\ldots+h_{n-1} x^{n-1}\left(h_{i} \in R[y]\right)$ is in the kernel of $\varphi \theta_{P}$ then we see from the first row of (1) that each $h_{i} \in P[y]$ and so $h \in P[x, \alpha]$.

Suppose $f \in R[x, \alpha]$ is nilpotent. Since any nilpotent $n \times n$ matrix $A$ over a field (or integral domain) satisfies $A^{n}=0$, and since $(R / P)[y]$ is an integral domain, we see that $\left(f \varphi \theta_{P}\right)^{n}=0$. Hence $f^{n}$ is in the kernel of $\varphi \theta_{P}$ which means that $f^{n} \in P[x, \alpha]$. Because $P$ was arbitrary, all coefficients of $f^{n}$ are in the prime radical of $R$ and so are nilpotent.

Conversely, consider any polynomial $r=r_{0}+r_{1} x+\ldots+r_{m} x^{m}\left(r_{i} \in R\right)$ such that all $r_{i}$ are nilpotent. If $r_{i}^{l}=0$ for all $i$, it follows that $r^{n l(m+1)}=0$, since a typical term in this is a product of $n t(m+1)$ terms $r_{i} x^{i}$ and so has coefficient a product of $n t(m+1)$ terms of the form $r_{i}^{\alpha^{j}}(0 \leqslant j \leqslant n-1)$, some $t$ of which must be equal. Thus if all coefficients of $f^{n}$ are nilpotent, $f^{n}$ is nilpotent and hence $f$ is nilpotent. 
Note. It follows from the second part of the above proof that $f$ is nilpotent if all its coefficients are nilpotent. That the converse is not true can be seen by taking $R=\mathbf{Z} \oplus \mathbf{Z}$ with $\alpha$ of order 2 given by $(a, b)^{\alpha}=(b, a)$. If

$$
f=(1,0) x+(1,-1) x^{2}+(0,-1) x^{3}
$$

then $f^{2}=0$ but the coefficient of $x^{2}$ in $f$ is a unit.

Nilpotent $n \times n$ matrices over a field are those with characteristic polynomial $\lambda^{n}$ and over a commutative ring are those with characteristic polynomial

$$
\lambda^{n}+d_{n-1} \lambda^{n-1}+\ldots+d_{1} \lambda+d_{0},
$$

where each $d_{i}$ is nilpotent. Hence the embedding $\varphi$ gives another description of the nilpotents.

Proposition 2. Let $R$ be a commutative ring with an automorphism $\alpha$ of order $n$. Then $f \in R[x, \alpha]$ is nilpotent if and only if $f \varphi$ has characteristic polynomial

$$
\lambda^{n}+d_{n-1} \lambda^{n-1}+\ldots+d_{1} \lambda+d_{0},
$$

where each $d_{i}$ is a nilpotent polynomial.

\section{Units}

THEOREM 3. Let $R$ be a commutative ring with identity and let $\alpha$ be an automorphism of $R$ with order $n$. A polynomial $f$ is a unit in $R[x, \alpha]$ precisely when the matrix $f \varphi$ has determinant $r_{0}+r_{1} y+\ldots+r_{m} y^{m}$ with $r_{0} a$ unit in $R$ and $r_{1}, \ldots, r_{m}$ nilpotent.

Proof. If $f$ is a unit in $R[x, \alpha]$ then clearly $f \varphi$ is a unit in $M(n, R[y])$. Since $R[y]$ is commutative, $\operatorname{det}(f \varphi)$ is a unit in $R[y]$ and hence has the appropriate form.

Conversely, if $\operatorname{det}(f \varphi)$ is as described, $\operatorname{det}(f \varphi)$ is a unit in $R[y]$ and so $f \varphi$ has an inverse $(f \varphi)^{-1}$ in $M(n, R[y])$. Because $f \varphi$ satisfies its characteristic polynomial,

$$
(f \varphi)^{-1}=(-1)^{n-1}(\operatorname{det} f \varphi)^{-1}\left((f \varphi)^{n-1}+c_{n-1}(f \varphi)^{n-2}+\ldots+c_{1}\right)
$$

for some $c_{1}, \ldots, c_{n-1} \in R[y]$. If

$$
g=(-1)^{n-1}(\operatorname{det} f \varphi)^{-1}\left(f^{n-1}+c_{n-1} f^{n-2}+\ldots+c_{1}\right)
$$

then $g \varphi$ has the same first row as $(f \varphi)^{-1}$ and so, since $(f \varphi)^{-1}(f \varphi)=I, g \varphi . f \varphi$ has first row $(1,0, \ldots, 0)$. Since $g \varphi . f \varphi=(g f) \varphi \in R[x, \alpha] \varphi$, it follows from (1) that $g \varphi . f \varphi=I$ and hence $g \varphi=(f \varphi)^{-1}$. Thus, since $\varphi$ is injective, $g f=f g=1$ and $f$ is a unit. 


\section{References}

R. Gilmer (1975), 'On polynomial and power series rings over a commutative ring', Rocky Mount. J. Math. 5, 157-175.

M. Rimmer (1978), Skew polynomial rings and skew power series rings (Ph.D. Thesis, La Trobe University).

La Trobe University

Bundoora, Victoria 3083

Australia 